\title{
Structure-Guided Creation of an Anti-HA Stalk Antibody F11 Derivative That Neutralizes Both F11-Sensitive and -Resistant Influenza A(H1N1)pdm09 Viruses
}

\author{
Osamu Kotani ${ }^{1, *, \dagger}$, Yasushi Suzuki ${ }^{2, \dagger}$, Shinji Saito ${ }^{3, *, \dagger}$, Akira Ainai $^{3}{ }^{3}$ Akira Ueno $^{3}$, Takuya Hemmi ${ }^{3}$, \\ Kaori Sano ${ }^{3}$, Koshiro Tabata ${ }^{3,4}$, Masaru Yokoyama ${ }^{1}$, , Tadaki Suzuki $^{3}{ }^{\circledR}$, Hideki Hasegawa ${ }^{2}$ and Hironori Sato ${ }^{1}$ \\ 1 Center for Pathogen Genomics, National Institute of Infectious Diseases, Tokyo 208-0011, Japan; \\ yokoyama@nih.go.jp (M.Y.); hirosato@nih.go.jp (H.S.) \\ 2 Center for Influenza and Respiratory Virus Research, National Institute of Infectious Diseases, \\ Tokyo 208-0011, Japan; yasuzuki@nih.go.jp (Y.S.); hasegawa@nih.go.jp (H.H.) \\ 3 Department of Pathology, National Institute of Infectious Diseases, Tokyo 162-8640, Japan; \\ ainai@nih.go.jp (A.A.); a-ueno@nih.go.jp (A.U.); t-hemmi@nih.go.jp (T.H.); ka-sano@nih.go.jp (K.S.); \\ k-tabata@czc.hokudai.ac.jp (K.T.); tksuzuki@nih.go.jp (T.S.) \\ 4 Division of Molecular Pathobiology, International Institute for Zoonosis Control, Hokkaido University, \\ Hokkaido 001-0020, Japan \\ * Correspondence: konioo@nih.go.jp (O.K.); ssaito@nih.go.jp (S.S.) \\ + Osamu Kotani, Yasushi Suzuki and Shinji Saito contributed equally to this work.
}

Citation: Kotani, O.; Suzuki, Y.; Saito, S.; Ainai, A.; Ueno, A.; Hemmi, T.; Sano, K.; Tabata, K.; Yokoyama, M.; Suzuki, T.; et al. Structure-Guided Creation of an Anti-HA Stalk Antibody F11 Derivative That Neutralizes Both F11-Sensitive and -Resistant Influenza A(H1N1)pdm09 Viruses. Viruses 2021, 13, 1733. https://doi.org/10.3390/v13091733

Academic Editor:

Stacey Schultz-Cherry

Received: 30 July 2021

Accepted: 26 August 2021

Published: 31 August 2021

Publisher's Note: MDPI stays neutral with regard to jurisdictional claims in published maps and institutional affiliations.

Copyright: () 2021 by the authors. Licensee MDPI, Basel, Switzerland. This article is an open access article distributed under the terms and conditions of the Creative Commons Attribution (CC BY) license (https:// creativecommons.org/licenses/by/ $4.0 /)$.

\begin{abstract}
The stalk domain of influenza virus envelope glycoprotein hemagglutinin (HA) constitutes the axis connecting the head and transmembrane domains, and plays pivotal roles in conformational rearrangements of HA for virus infection. Here we characterized molecular interactions between the anti-HA stalk neutralization antibody F11 and influenza A(H1N1)pdm09 HA to understand the structural basis of the actions and modifications of this antibody. In silico structural analyses using a model of the trimeric HA ectodomain indicated that the F11 Fab fragment has physicochemical properties, allowing it to crosslink two HA monomers by binding to a region near the proteolytic cleavage site of the stalk domain. Interestingly, the F11 binding allosterically caused a marked suppression of the structural dynamics of the HA cleavage loop and flanking regions. Structureguided mutagenesis of the F11 antibody revealed a critical residue in the F11 light chain for the F11-mediated neutralization. Finally, the mutagenesis led to identification of a unique F11 derivative that can neutralize both F11-sensitive and F11-resistant $\mathrm{A}(\mathrm{H} 1 \mathrm{~N} 1)$ pdm09 viruses. These results raise the possibility that F11 sterically and physically disturbs proteolytic cleavage of HA for the ordered conformational rearrangements and suggest that in silico guiding experiments can be useful to create anti-HA stalk antibodies with new phenotypes.
\end{abstract}

Keywords: influenza virus; anti-HA stalk antibody; molecular interactions; molecular dynamics simulation; antibody modification; neutralization assay

\section{Introduction}

Infection with the influenza virus often causes serious respiratory diseases in humans, and thus presents a persistent public health threat. Although vaccination is a promising strategy to suppress the magnitude of an influenza epidemic, current vaccines rarely overcome the genetic diversity of influenza viruses. The major immunodominant region for raising neutralization antibodies is located in a highly variable head domain of envelope glycoprotein hemagglutinin (HA) [1-4]. Consequently, neutralization antibodies raised against the head domain are usually strain-specific, and often drive the selection of new variants that are less sensitive to the circulating antibodies [5]. Therefore, antibodies that target more conserved regions have been sought to combat infection by influenza viruses [6-8]. 
Monoclonal antibodies that target the stalk domain of the HA protein have been shown to protect mice against a broad range of influenza viruses [9]. Moreover, viruses that acquired resistance to the anti-HA stalk antibodies were attenuated in mice and could be controlled with a candidate vaccine [10]. The stalk domain is located in an extracellular portion of the HA protein, and is a key structural unit for the $\mathrm{pH}$-dependent conformational changes of the HA protein to gain membrane fusion activity for genome uncoating in the endosome, thereby playing essential roles in establishing virus infection in the cells [11]. The stalk domain is relatively more conserved within and among influenza virus strains in nature, as compared with the HA globular region [2,4]. This may imply that the stalk domain is under strong functional/structural constraints against changes. Thus, antibodies targeting the stalk domain can be beneficial tools to combat infections of highly mutable influenza viruses [6-8]. To date, many anti-HA stalk antibodies have been isolated and characterized for their binding modes to HA protein [9,12-17]. Interestingly, about half of these antibodies bind to HA in a manner that crosslinks two HA monomers of the HA trimer on the virion $[9,12-14]$. Based on the structural information, the anti-HA stalk antibodies have been proposed to inhibit conformational changes of the HA protein that establish the infections of target cells [9,12-17].

Recently, we independently isolated an anti-HA stalk antibody, termed F11, from an individual inoculated with an intranasal inactivated influenza vaccine [18]. In its IgA form, F11 was able to neutralize the H1N1, H3N2, and H5N1 strains of influenza A, but in its IgG form it had lower cross-reactivity [18]. We also isolated variants of influenza A(H1N1)pdm09 that exhibited reduced susceptibilities to neutralization mediated by the F11 antibody [18]. We found that single substitutions near the hydrophobic cleft of the HA stalk region could confer F11 resistance to the virus in association with changes in the physicochemical properties of the hydrophobic cleft [18]. However, the binding mode of F11 to HA and therefore the molecular mechanisms underlying the F11-mediated neutralization remain unknown. To address each of these issues, we herein examined the structural basis of molecular interactions between the F11 Fab fragment and glycosylated HA trimer using in silico techniques involving molecular dynamics (MD) simulations. We then used the obtained information to create a F11 variant that can neutralize both F11-sensitive and F11-resistant influenza A(H1N1)pdm09 viruses.

\section{Materials and Methods}

\subsection{Molecular Modeling of the Trimeric HA Ectodomain of Influenza A(H1N1)pdm09}

A three-dimensional model of the HA trimer ectodomain of the A/Narita/1/2009 (H1N1)pdm09 virus in the ligand-free state was first constructed by the homology modeling method using the reported amino acid sequence of HA of A/Narita/1/2009 (H1N1)pdm09 [19] (Figure S1). The Molecular Operating Environment (MOE) (Chemical Computing Group Inc., Montreal, QC, Canada) was used for the homology modeling. A high resolution crystal structure of an HA trimer of the A/California/04/2009 (H1N1) virus (PDB code: 3LZG, a resolution of $2.6 \AA$ ) [20] was used as the modeling template. The template represents a structure of the extracellular portion of the H1N1 HA strain. The highmannose oligosaccharide $\mathrm{Man}_{5} \mathrm{GlcNAc}_{2}$ was attached to potential $\mathrm{N}$-glycosylation sites in HA using tools in GLYCAM-Web [21]. Subsequently, the glycosylated HA trimer model was subjected to MD simulation as described for HA trimers of influenza A virus [18,22]. Briefly, the simulations were performed using the pmemd.cuda.MPI module in the Amber 16 program package [23] with the ff14SB force field for simulation of protein [24], with the GLYCAM06j-1 force field for simulation of glycan [25], and with the TIP3P water model for simulation of aqueous solutions [26]. A non-bonded cut-off of $10 \AA$ was used. Bond lengths involving hydrogen were constrained with SHAKE, a constraint algorithm that satisfies Newtonian motion [27]. The time step for all MD simulations was set to $2 \mathrm{fs}$. After heating calculations performed for $20 \mathrm{ps}$ up to $310 \mathrm{~K}$ using the NVT ensemble, simulations were executed for $400 \mathrm{~ns}$ in $150 \mathrm{mM} \mathrm{NaCl}$ using the NPT ensemble (at $1 \mathrm{~atm}, 310 \mathrm{~K}$ ). 


\subsection{Docking Simulations of the Glycosylated HA Ectodomain and Anti-HA Stalk Antibody F11}

A three-dimensional model of the stalk antibody F11 Fab fragment was constructed using the Antibody Modeler application of MOE [28]. Amino acid sequences of the antibody F11 immunoglobulin light chain (GenBank accession number: QII15722) and heavy chain (GenBank accession number: QII15721) were used for modeling of the Fab fragment (Figure S1A) [18]. The obtained models were optimized by energy minimization using the Amber10: Extended Huckel Theory (EHT) force field implemented in MOE. Docking simulations were performed using the Dock application of MOE $[29,30]$. Briefly, amino acid residues in the HA stalk domain (HA1: 18-57; HA2: 345-515) (Figure S1B) from a structure of the glycosylated HA trimer at $400 \mathrm{~ns}$ of MD simulation were defined as a receptor for binding of stalk-antibody F11, while an energy minimized structure of a variable region of the F11 Fab fragment was defined as a ligand for binding to the HA stalk domain of the glycosylated-HA trimer. Subsequently, physicochemically possible docking poses between the receptor and ligand moieties were comprehensively searched with the Dock application. The docking simulations were executed to collect 100 possible docking poses. Binding energies of the F11 Fab fragment to the glycosylated-HA trimer ectodomain were calculated with the Dock tool using individual docking poses.

\subsection{Simulation of the Glycosylated HA Trimer Docked to the F11 Fab Fragment}

The complex model obtained by in silico docking simulation was subjected to MD simulation under the conditions described previously with HA trimers of influenza A virus [18,22], which were essentially the same as the conditions used for MD simulations of HIV-1 structural proteins such as capsid [31] and envelope gp120 bound to CD4 and CCR5 [32]. Briefly, MD simulations were performed using the pmemd.cuda.MPI module in the Amber 16 program package [23] with the ff14SB force field for simulation of protein [24], with the GLYCAM06j-1 force field for simulation of glycan [25] and with the TIP3P water model for simulation of aqueous solutions [26]. A non-bonded cut-off of $10 \AA$ was used. Bond lengths involving hydrogen were constrained with SHAKE, a constraint algorithm that satisfies Newtonian motion [27]. The time step for all MD simulations was set to $2 \mathrm{fs}$. After heating calculations performed for $20 \mathrm{ps}$ up to $310 \mathrm{~K}$ using the NVT ensemble, simulations were executed for $200 \mathrm{~ns}$ in $150 \mathrm{mM} \mathrm{NaCl}$ using the NPT ensemble (at $1 \mathrm{~atm}, 310 \mathrm{~K}$ ).

\subsection{Calculation of Root Mean Square Deviation (RMSD)}

Calculations of RMSDs were done as described previously [32,33]. Briefly, RMSDs between the heavy atoms of the initial complex structure and the structures at given time points of the MD simulation were calculated to monitor the overall structural changes during the MD simulations using the cpptraj module in AmberTools 16 [23].

\subsection{Calculation of Root Mean Square Fluctuation (RMSF)}

The trajectory files during last $20 \mathrm{~ns}$ of MD simulations $(n=10,000)$ were used to calculate RMSF. RMSF of the C $\alpha$ atoms of amino acid residues was calculated to obtain information regarding the atomic fluctuations of individual amino acid residues of HA protein during MD simulations as described previously [18,31-33] using the tools in Amber 16 [23]. Briefly, the average structures during the last $20 \mathrm{~ns}$ of MD simulations were used as reference structures for RMSF calculation. RMSF, which quantifies differences between average values and those obtained at a given time point of MD simulation, was calculated using the ptraj module, a trajectory analysis tool in AmberTools 16 [23].

\subsection{Analysis of Noncovalent Interaction Sites and Networks between HA and F11}

Analyses of noncovalent intermolecular interactions for F11 antibody binding to the HA protein during MD simulations were performed as described previously [33]. Briefly, noncovalent interactions between the F11 antibody and HA protein were identified using the Contact Analysis application of MOE. This application allows identification of distance-dependent noncovalent interactions between atoms, such as hydrogen bonds, 
Van der Waals interactions, $\pi$-interactions, and ionic interactions. HA-F11 complex structures $(n=20)$ collected at every $1 \mathrm{~ns}$ during 180 to $200 \mathrm{~ns}$ after MD simulations were used for the search of interaction sites. Noncovalent interaction networks in the HA/F11 complex detected in the 20 complex structures were visualized using Cytoscape software version 3.8.2 [34], a program for integrated models of biomolecular interaction networks.

\subsection{Protein Patch Analysis}

Assessment and visualization of hydrophobic patches on the HA-F11 complex model were done as described previously [31] using the Protein Patch Analyzer tool in MOE [35-38]. Briefly, protein hydrophobic patches with a minimum area of $50 \AA^{2}$ for protein-protein interactions $[39,40]$ were identified with the HA-F11 complex structure at $200 \mathrm{~ns}$ of MD simulation using the Protein Patch Analyzer.

\subsection{Shannon Entropy Analysis}

Amino acid variation at contact sites of the HA protein was analyzed with Shannon entropy as described previously [31,41]. Amino acid sequences of the HA protein of the influenza A virus $\mathrm{H} 1$ ( $n=36,564$; former seasonal H1N1 and H1N1pdm) and $\mathrm{H} 3(n=53,989$; H3N2 and H3N2v) were obtained from the GISAID database [42]. Shannon entropy was calculated on the basis of Shannon's equation [43]:

$$
H(i)=-\sum_{x_{i}} p\left(x_{i}\right) \log _{2} p\left(x_{i}\right) \quad\left(x_{i}=G, A, I, V, \ldots\right)
$$

where $H(i), p\left(x_{i}\right)$, and $i$ indicate the amino acid entropy score for an individual position, the probability of occurrence of a given amino acid at that position, and the number of the position, respectively. An $H(i)$ score of zero indicates absolute conservation, whereas a score of 4.4 bits indicates complete randomness.

\subsection{In Silico Site-Directed Mutagenesis of the F11 Antibody}

F11-HA complex structures obtained during 180 to 200 ns after MD simulations were used for site-directed mutagenesis of the F11 antibody. For the comprehensive mutagenesis study, F11 residues forming noncovalent bonds with HA glycan or amino acid were identified with the structures during 180 to $200 \mathrm{~ns}$ of MD simulation using the Contact Analysis application of MOE. Subsequently the contact residues and surrounding residues of F11 were individually substituted with 19 nonself amino acid residues. Modeling of the F11 mutants and calculation of changes in the F11 stability and binding affinity to HA by the mutations were done using the Protein Design application of MOE, along with the MM/GBVI program to calculate the binding affinity as described previously [44-46]. The results were expressed by the change in the free energy of binding $(\Delta \Delta \mathrm{G})$. In some cases, binding energies of individual complexes of F11-HA obtained during MD simulations were calculated using the Potential Energy application of MOE to assess the distribution of binding energies in solution.

\subsection{Cells and Viruses}

Madin-Darby canine kidney (MDCK) cells (American Type Culture Collection; CCL34) were maintained at $37{ }^{\circ} \mathrm{C} / 5 \% \mathrm{CO}_{2}$ in minimum essential medium (MEM; Life Technologies, Carlsbad, CA, USA) containing 10\% fetal bovine serum (Thermo Fisher Scientific, Waltham, MA, USA) and pen-strep mix (100 units/mL penicillin and $100 \mu \mathrm{g} / \mathrm{mL}$ streptomycin; Life Technologies). Humanized MDCK (hCK) cells [47] were maintained at $37^{\circ} \mathrm{C} / 5 \%$ $\mathrm{CO}_{2}$ in Dulbecco's Modified Eagle's Medium-high glucose (DMEM; Sigma-Aldrich, St. Louis, MO, USA) containing $5 \%$ fetal bovine serum, pen-strep mix (100 units/mL penicillin and $100 \mu \mathrm{g} / \mathrm{mL}$ streptomycin), $5 \mu \mathrm{g} / \mathrm{mL}$ blasticidin (InvivoGen, San Diego, CA, USA), and $2 \mu \mathrm{g} / \mathrm{mL}$ puromycin dihydrochloride (Life Technologies). Expi293F cells (Thermo Fisher Scientific) were maintained at $37^{\circ} \mathrm{C} / 8 \% \mathrm{CO}_{2}$ in Erlenmeyer cell culture flasks (Corning, Tewksbury, MA, USA) containing Expi293 Expression Medium (Thermo Fisher Scientific). 
A/Narita/1/2009 (H1N1)pdm09 virus [19] and A/Narita/1/2009 (H1N1)pdm09-derived F11 escape mutants (C1 and G6 [18]) were propagated in MDCK cells.

\subsection{Site-Directed Mutagenesis, Expression, and Purification of IgG Antibodies}

Plasmids encoding F11 $\gamma 1 \mathrm{HC}$ and F11 $\mathrm{kLC}$ were prepared as previously described [18]. DNA fragments encoding the variable region of the heavy or light chain of each mutant F11 clone were Homo sapiens codon-optimized and synthesized by the GenPlus Highthroughput Gene Synthesis service (GenScript, Piscataway, NJ, USA). Synthesized DNA fragments were cloned into $\gamma 1 \mathrm{HC}$ or $\mathrm{kLC}$ vectors and used for the site-directed mutagenesis. To generate IgG1, Expi293F cells grown in Expi293 Expression Medium were diluted to $2.5 \times 10^{6}$ cells $/ \mathrm{mL}$ and transfected with $26 \mu \mathrm{g}$ of $\gamma 1 \mathrm{HC}$ and $24 \mu \mathrm{g}$ of $\mathrm{kLC}$ per $50 \mathrm{~mL}$ of final culture volume using the ExpiFectamine 293 Transfection Kit (Thermo Fisher Scientific). At 7 days post-transfection, cell culture supernatants were centrifuged at $1200 \times g$ and filtered to remove cell debris. The supernatants were then purified using CaptureSelect CH1-XL (Thermo Fisher Scientific) according to the manufacturer's instructions. Purified antibodies were concentrated using Amicon Ultracell (Merck, Darmstadt, Germany) centrifugation units with a cut-off of $30 \mathrm{kDa}$; the buffer was changed to $20 \mathrm{mM}$ phosphate buffer (pH 7.4) using a Zeba Spin Desalting Column (Thermo Fisher Scientific).

\subsection{Assessment of Mutated IgG1 Antibody Quality by SDS-PAGE Analysis}

To confirm successful production and purification of mutated IgG1 antibodies, purified antibodies were analyzed by SDS-PAGE on $5-20 \%$ gradient e-PAGEL gel (ATTO Inc., Tokyo, Japan). Precision Plus Protein Dual Color Standards (Bio-Rad, Hercules, CA, USA) were used as molecular weight markers. The SDS-PAGE gels were stained with QuickCBB PLUS (FUJIFILM Wako Pure Chemical Corporation, Osaka, Japan) according to the manufacturer's instructions.

\subsection{Microneutralization (NT) Assay}

The microneutralization assay was performed using hCK cells [47] and $100 \mathrm{TCID}_{50}$ of influenza virus, essentially as previously described [48,49]. Briefly, each sample (serially diluted 2-fold) was mixed with an equal volume of diluent containing influenza virus (equivalent to $100 \mathrm{TCID}_{50}$ ) and incubated for $30 \mathrm{~min}$ at $37^{\circ} \mathrm{C}$. This mixture was added to a monolayer of hCK cells in the wells of a 96-well plate. Four control wells containing virus or diluent alone were included on each plate. The plates were incubated for 5 days at $37{ }^{\circ} \mathrm{C} / 5 \% \mathrm{CO}_{2}$. All wells were observed for the presence or absence of cytopathic effects under light microscopy, fixed with $10 \%$ formalin phosphate buffer for more than $5 \mathrm{~min}$ at room temperature, and then stained with Naphthol blue black. After washing and drying, cells were solubilized with $0.1 \mathrm{M} \mathrm{NaOH}$ and absorbance (A) was read at $630 \mathrm{~nm}$. The average A630 value was determined from virus-only controls (A virus) and medium only controls (A cell). Values $>50 \%$ of the specific signal, calculated using the formula $X=($ A cell $-\mathrm{A}$ virus $) / 2$, were considered positive for neutralization. NT activity was defined as the reciprocal of the lowest concentration $(\mu \mathrm{g} / \mathrm{mL})$ of antibody, at which point $\mathrm{A} 630$ was $>\mathrm{X}$.

\subsection{Statistical Analysis}

All statistical analyses were performed using the Prism statistical software package (version 7.0; GraphPad Software). An ordinary one-way ANOVA followed by Dunnett's multiple comparison test or a Kruskal-Wallis test followed by Dunn's multiple comparison test was used to analyze each dataset, as indicated in the figure legends. The threshold for statistical significance was set at $5 \%(p<0.05)$. 


\section{Results}

\subsection{Molecular Modeling of the Glycosylated HA Trimer Ectodomain Docked to the F11 Fab Fragment}

To conduct an in silico structural study of the interactions between the F11 antibody and HA, we constructed a molecular model of the glycosylated HA trimer ectodomain docked to the F11 Fab fragment using homology modeling, molecular dynamics (MD) simulation, and docking simulation as described in Materials and Methods. We first constructed a model of the unliganded HA trimer ectodomain of the A/Narita/1/2009 (H1N1)pdm09 virus [19] used in a previous report [18] and the present study by homology modeling. The high-mannose oligosaccharide $\mathrm{Man}_{5} \mathrm{GlcNAc}_{2}$ was added to the potential $N$-glycosylation sites in the trimeric HA using tools in GLYCAM-Web [21].

To obtain trimeric HA ectodomain structures in solution, the initial model was subjected to MD simulation. The structural changes after the start of MD simulation were monitored with root mean square deviation (RMSD) [23] between the initial model structure and the structures at given time points of the MD simulation as described for HA trimers of influenza A virus [18,22] (Figure 1A). The RMSDs sharply increased in the beginning and reached a near plateau after $20 \mathrm{~ns}$ of the MD simulations. The results suggest that the structural distortions in the initial HA trimer model were relieved shortly after the onset of MD simulation, and reached a state of thermodynamic equilibrium in solution. The MD simulation also disclosed that glycans at the HA stalk domain had partially hidden the hydrophobic groove [50] located near the binding interface of the reported anti-HA stalk antibodies $[9,12-14,51,52]$ (Figure 1B). These results suggest that the HA ectodomain in solution preserves a structure that is partially protected against anti-HA stalk antibodies by the HA stalk glycans.

Using the HA trimer model in the equilibrium state under solution conditions, we conducted in silico docking simulation between HA and the F11 Fab fragment (Figure 1C). The distribution of the free energy of binding $(\Delta G)$ showed that various docking poses were physicochemically possible. Among the top 100 docking poses, the vast majority of F11 Fab was bound to a region near the hydrophobic groove [50], in the same manner that anti-HA stalk antibodies were previously shown to bind to the trimeric HA ectodomain [9,12-14,51,52] (Figure 1D). We then examined individual docking poses. The highest ranked binding mode (Figure 1C, top-1 binding mode) was unlikely to reflect the natural binding mode of the antibody, since the constant region of Fab was involved in the HA binding and the binding mode could cause a steric clash between the HA head domain and F11 FC region (Figure S2A). In contrast, only the variable domains of F11 participated in the HA binding and no steric crush was predicted to occur in the binding mode with the second highest binding affinity (Figure 1E and Figure S2B, top-2 binding mode). The top-2 binding mode featured the crosslinking of two HA monomers by F11 Fab variable regions (Figure 1E). The top-2-like binding modes involving HA crosslinking were most frequently seen among the 100 docking poses (Figure S2C). These results suggest that the F11 Fab fragment has physicochemical properties that crosslink two HA monomers by binding to the stalk domain of the glycosylated HA trimer ectodomain.

\subsection{Simulation of Glycosylated HA Trimer Docked to F11 Fab Fragment}

To assess the stability of the HA-F11 Fab complex, we ran an MD simulation using the top-2 binding mode (Figure 2). The RMSDs sharply increased after the onset of the MD simulations, reaching a near plateau after $100 \mathrm{~ns}$, but with greater levels of fluctuations as compared with those of the unliganded HA trimer (Figures 1A and 2A). This structural fluctuation mainly reflected that of the F11 Fab linker region between the variable region (VR) and constant region (CR), and fluctuations of the VR for HA binding were minimal, if any (Figure 2B). Consequently, no major shifting in the binding site was detected before and after the MD simulation (Figure 2C). These results suggest that the top-2 binding mode is thermodynamically stable under solution conditions. 
A

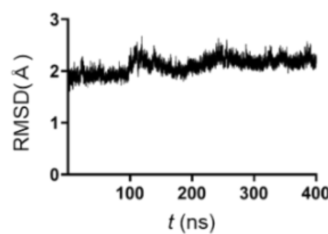

B

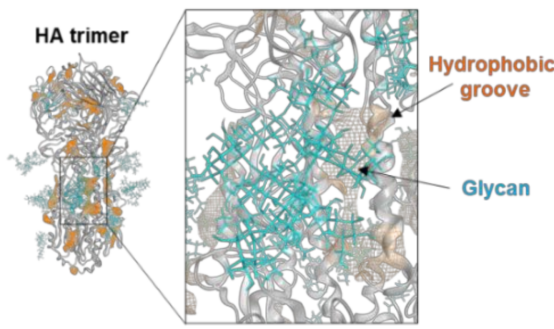

C

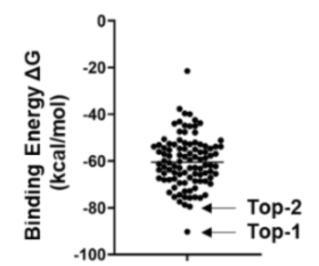

D

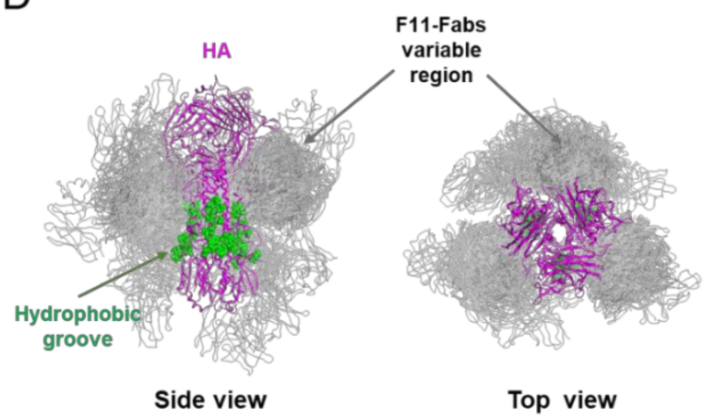

E

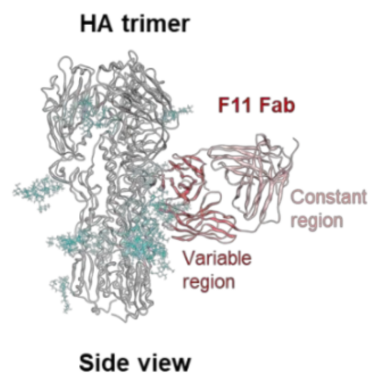

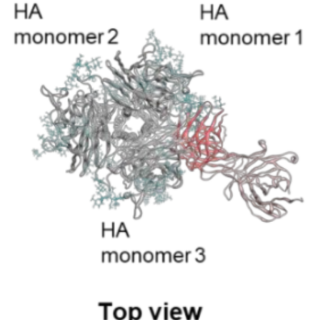

Figure 1. Molecular modeling of the complex of glycosylated HA trimer and F11 Fab fragment. A three-dimensional model of the unliganded, glycosylated HA trimer ectodomain of A/Narita/1/2009 (H1N1)pdm09 virus [19] was constructed by the homology modeling method using the Molecular Operating Environment (MOE) (Chemical Computing Group Inc., Montreal, QC, Canada), glycosylated using tools in GLYCAM-Web [21], and subjected to MD simulation using modules in the Amber 16 program package [23]. The HA trimer model in the equilibrium state under solution conditions was used for the docking simulations of the F11 Fab fragment using the Dock application of MOE [29,30]. (A) Root mean square deviation (RMSD) [23] between the structure of the initial HA structure and those at given time points of the MD simulation of glycosylated HA trimers calculated using the cpptraj module in AmberTools 16 as described previously [22]. (B) Side view of the HA trimer model at $400 \mathrm{~ns}$ of MD simulation. (C) Distribution of binding energies among the top 100 binding poses generated using the Dock application of MOE [29,30]. (D) Superposition of 100 docking poses. The purple region indicates the HA trimer. Green regions indicate the hydrophobic groove [50] in the HA trimer [53,54]. Grey regions indicate F11 Fab variable regions of the top 100 docking poses. Glycans attached on the HA trimer are not shown here in order to highlight the binding mode of the F11 Fab variable region. (E) The second highest ranked binding mode of F11 Fab (top-2 binding mode). Cyan branches indicate the high-mannose oligosaccharide $\mathrm{Man}_{5} \mathrm{GlcNAc}_{2}$.

\subsection{Characterization of Molecular Interactions between the HA Trimer Ectodomain and F11 Fab Fragment}

The above results suggest that the top-2 complex represents a biologically, physically, and thermodynamically favorable binding mode with the highest binding affinity to the HA ectodomain. Therefore, we further examined details of the molecular interactions using the top-2 structures derived from MD simulation. HA-F11Fab complexes during 180 to $200 \mathrm{~ns}$ of MD simulations were used to identify noncovalent interactions between the two molecules (Figure 3). The binding interface of HA during MD simulations consisted of 16 amino acid residues near the hydrophobic groove [50], the proteolytic cleavage site, and the fusion peptide (Figure 3A, left panel). The binding surface of F11 during MD simulations consisted of 20 amino acid residues in the complementarity determining region (CDR) of the heavy and light chains (Figure 3A, right panel). Interactions at individual contact sites could be classified into three groups (Figure 3B): (1) those between three glycans in the HA stalk domain and CDR of F11 heavy/light chains; (2) those between HA and the F11 light chain; and (3) those between HA and the F11 heavy chain. Closely overlapping hydrophobic regions existed around I341 in the HA proteolytic cleavage loop and F11 I30 in the light chain CDR1 region (Figure 3C). These data suggest that the F11 Fab fragment could bind to trimeric HA via long-range interactions near the functional sites of the HA stalk. 


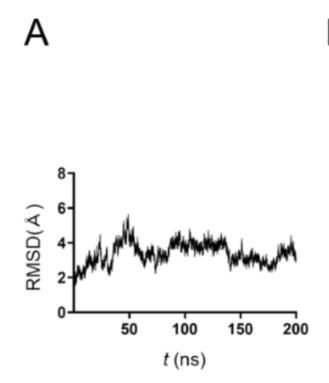

B

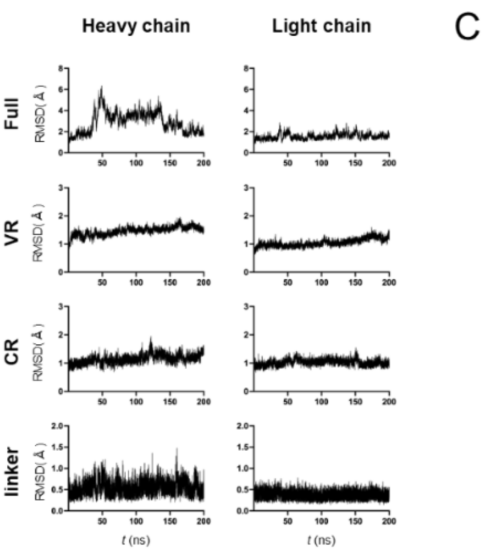

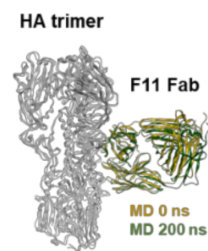

Side view

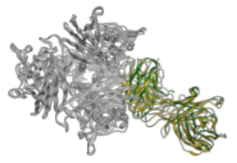

Top view

Figure 2. MD simulation of glycosylated HA trimer docked to the F11 Fab fragment. The top-2 complex was subjected to MD simulation at $1 \mathrm{~atm}$ and at $310 \mathrm{~K}$ in $0.15 \mathrm{M} \mathrm{NaCl}$ for $200 \mathrm{~ns}$. (A) RMSDs between the structure of the initial HA-F11 Fab complex and those at the given time points of the MD simulation are shown. (B) RMSDs of structural units of the F11 Fab fragments during the MD simulations. The entire region (Full), variable region (VR), constant region (CR), and unstructured linker region between VR and CR (linker) are shown for the heavy chain (left panels) and light chain (right panels). (C) Superposition of the top-2 complex structures before and after $200 \mathrm{~ns}$ of MD simulations.

A

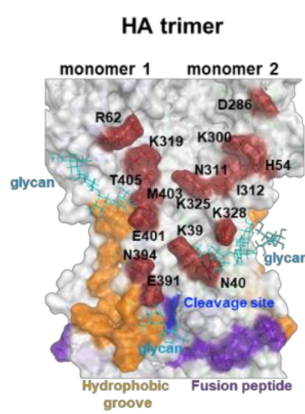

C

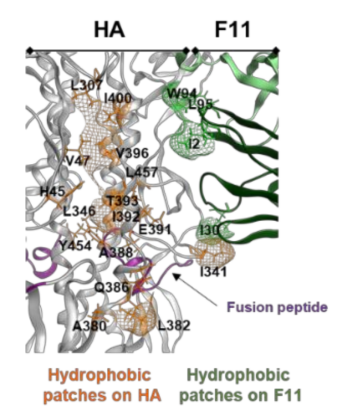

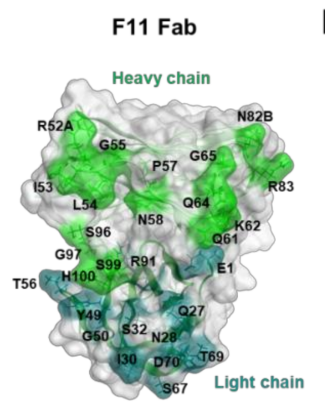

B

D

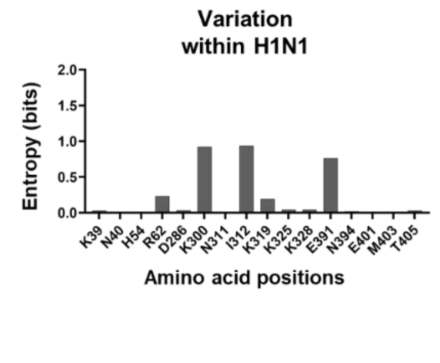

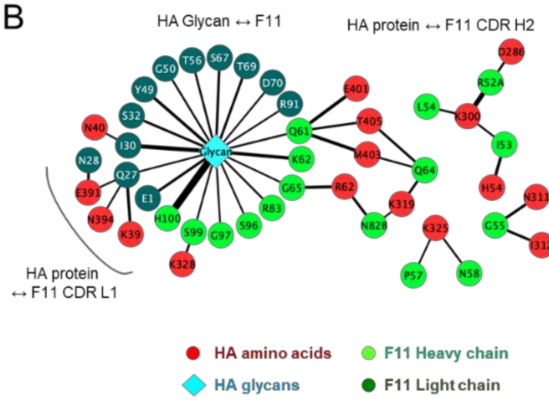

Variation among H1N1 and H3N2

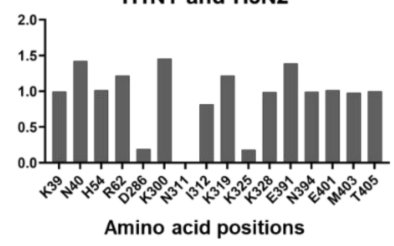

Figure 3. Characterization of molecular interactions between the HA trimer ectodomain and F11 Fab fragment. HA-F11 complex structures collected at every 1 ns during 180 to $200 \mathrm{~ns}$ after MD simulations of the top-2 binding mode were used to search for the sites supporting molecular interactions between HA and the F11 antibody using the Contact Analysis application of MOE. (A) Visualization of contact sites in the HA trimer (left, wine red residues) and F11 Fab variable region (right, light green and blue green). (B) Interaction networks between HA and F11 Fab variable regions. Non-covalent interactions detected during MD simulation were visualized using Cytoscape software version 3.8.2 [34] as described previously [33]. (C) Distribution of hydrophobic patches in the binding interface of HA and F11. Hydrophobic patches with a minimum area of $50 \AA^{2}$ for protein-protein interactions were estimated using the Protein Patch Analyzer tool in MOE as described previously [31]. (D) Variation of individual amino acid residues forming noncovalent interactions during MD simulation. Amino acid sequences of the HA protein of the influenza A virus H1 $(n=36,564)$ and H3 $(n=53,989)$ were obtained from the GISAID database [42]. Shannon entropy [43] was calculated for individual contact sites detected during MD simulation as described previously [31,41].

HA stalk amino acid residues constituting the contact sites were basically highly conserved within influenza A/H1 viruses except for a few sites (Figure 3D, left panel), 
whereas they were markedly different between the influenza A/H1 and A/H3 viruses (Figure 3D, right panel). These results suggest that the F11 antibody is primarily effective on $\mathrm{A} / \mathrm{H} 1$ viruses but not on $\mathrm{A} / \mathrm{H} 3$ viruses. This prediction is consistent with the previous finding that the F11 IgG antibody effectively blocked infections by A/H1 viruses, but not $\mathrm{A} / \mathrm{H} 5$ viruses [18].

\subsection{Effects of F11 Fab Binding on the Structural Fluctuations of the HA Ectodomain}

Structural fluctuations of biological macromolecules in solution play key roles in molecular interactions [55-58] and thus in the biological phenotypes of viruses [18,31-33]. To gain insights into the structural impact of F11 binding to HA, we compared fluctuations of the HA ectodomain between the free and F11-bound forms using root mean square fluctuation (RMSF), a quantitative indicator of atomic fluctuation [23] (Figure 4). As expected from previous reports $[18,31,32]$, structural fluctuations were often seen in the loops of the ligand-free HA glycoprotein (Figure 4A, free HA). Notably, the proteolytic cleavage loop in the stalk domain, which is the target of cellular proteases [59-62], was found to fluctuate most heavily in the HA ectodomain under solution conditions. In contrast to the marked impacts on the HA cleavage site, F11 binding to the HA ectodomain did not affect fluctuations in the regions for the HA receptor binding in the head domain [63,64] (Figure 4A, 130 loop, 190 helix, and 220 loop). Interestingly, F11 binding to HA markedly reduced the structural fluctuations around the cleavage loop, as indicated by the marked reduction in the RMSF values (Figure 4A, F11-bound HA). The suppression was detected in the residue at position 327 (T327), residues in the cleavage loop, and residues in the fusion peptide for membrane fusion [50] (Figure 4A). It should be noted that these regions were not direct contact sites of F11, except for the T327 neighboring of an F11 contact residue K328 (Figures 3A and 4B). Instead, the regions that were influenced by the F11 binding were basically assembled to lie within the F11 binding sites (Figure 4B). These results suggest that F11 binding could structurally and physically alter the conformational rearrangement of the HA ectodomain.

\subsection{In Silico Site-Directed Mutagenesis}

To assess the relative contributions of individual contact residues of F11 to the binding affinity of F11 Fab to the HA stalk domain, we conducted in silico site-directed mutagenesis of the contact sites. The F11 Fab residues that were capable of interacting noncovalently with HA (Figure 3A) and the surrounding residues were individually exchanged with 19 nonself residues, and their effects on F11 stability and activity were analyzed as described previously [44-46] (Figure 5). The single substitutions tested had little effect on the stability of the F11 Fab fragment (Figure 5A), as expected from the fact that the substitutions were introduced in the variable region of the antibody. In contrast to the stability, the F11 substitutions had some impact on the calculated binding affinity of Fab to HA, although these effects were minor, mostly consisting of changes in the free energy of binding $(\Delta \Delta \mathrm{G})$ within $5 \mathrm{kcal} /$ mole or less (Figure 5B).

We chose eight single substitutions for further analyses. These F11 substitutions presumably had a different influence on the binding affinity to the HA ectodomain without affecting the F11 stability (Figure 5C,D). Single substitutions at positions 64 and 65 (Q64W, G65F, and G65Q) in the F11 heavy chain, which noncovalently interacted with HA residues near the upper part of the hydrophobic groove during MD simulation (Figure 3A,B), were predicted to augment the binding affinity (Figure 5D). G65P substitution was predicted to cause no major changes in the binding affinity, suggesting that the types of side chain at position 65 are important to maintain the binding affinity. Substitutions at position 93 in the F11 light chain, which exhibited no noncovalent interactions with HA during MD simulation, somehow augmented the binding affinity. In contrast to these findings, single substitutions at position 49 in the F11 light chain, which noncovalently interacted with glycan on asparagine at position 40 (N40) near the cleavage site of HA (Figure 3A,B), were predicted to attenuate the binding affinity (Figure 5D). 
A

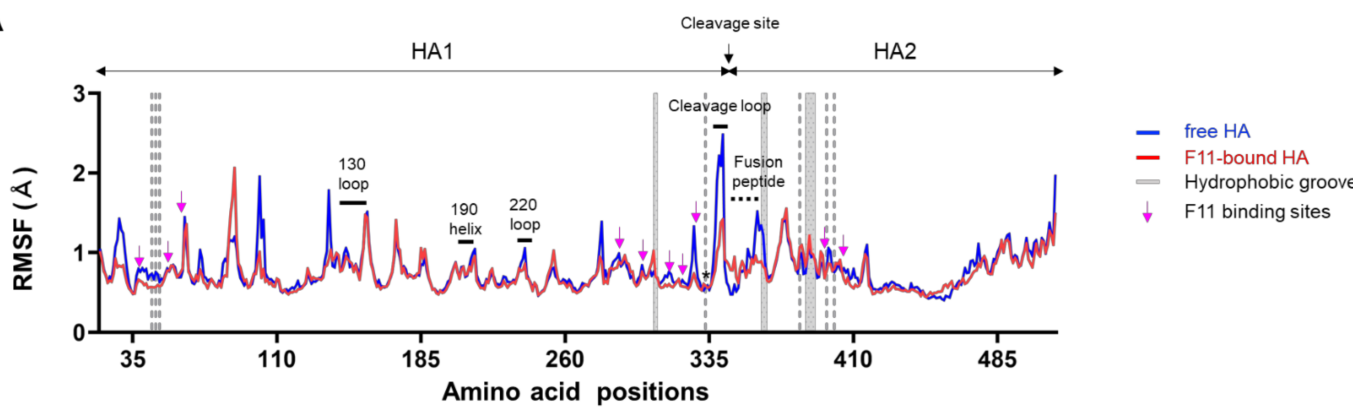

B

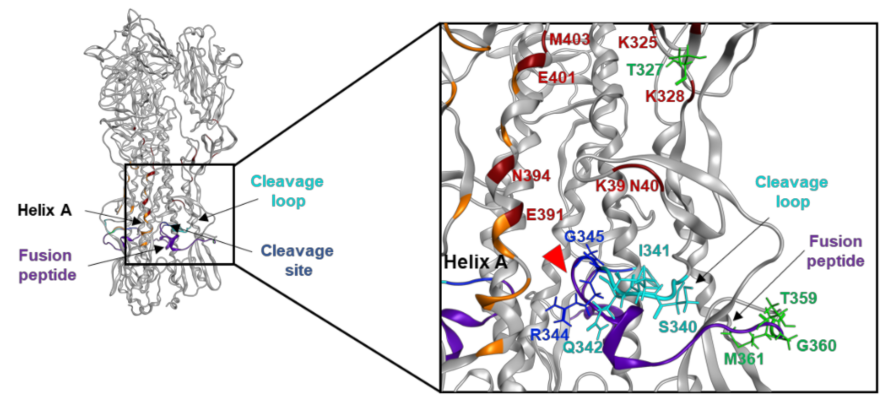

Figure 4. Effects of F11 Fab binding on the structural fluctuations of the HA ectodomain. Free and F11 Fab-bound trimeric HA ectodomains were subjected to MD simulations for $200 \mathrm{~ns}$ using the Amber 16 program package [23]. RMSF values of the $\mathrm{C} \alpha$ atoms of individual HA residues were calculated using 10,000 snapshots during 180 to $200 \mathrm{~ns}$ of MD simulations as described previously [18,31,32]. (A) Distributions of RMSF in HA. Numbers on the horizontal axes indicate positions in the HA of influenza A/Narita/1/2009 (H1N1)pdm09 [19]. Magenta arrows indicate the residues contacting F11 Fab during 180 to $200 \mathrm{~ns}$ of MD simulations of the top-2 binding mode. The gray shadows indicate locations of HA residues constituting the hydrophobic groove. Regions involved in sialic acid binding [63,64], such as the 130 loop, 190 helix, and 220 loop, are merged with black bars. (B) Structure around the proteolytic cleavage loop. The cleavage loop, fusion peptide [64], and hydrophobic groove $[53,54]$ are shown in cyan, purple, and orange, respectively. A red arrowhead indicates the cleavage site. Residues that noncovalently interacted with the F11 Fab fragment during MD simulation are marked with a wine red color.

A

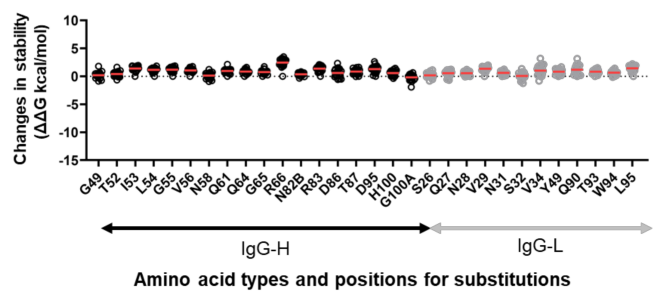

B

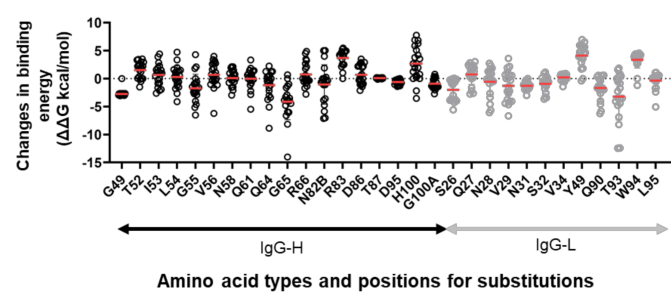

C

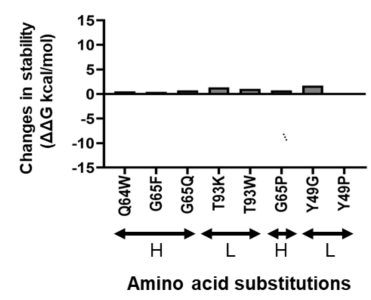

D

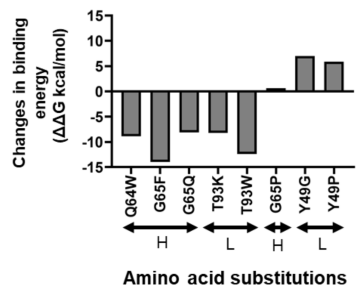

Figure 5. In silico site-directed mutagenesis. F11 Fab residues capable of noncovalently interacting with HA (Figure 3A) and the surrounding residues were individually exchanged for 19 nonself residues, and changes in the stability and binding affinity of F11 Fab were calculated using the F11/HA complex structure obtained at $200 \mathrm{~ns}$ of MD simulations as described previously [44-46] using the Protein Design application of MOE. (A,C) Effects on the stability of the F11 Fab fragment. (B,D) Effects on the binding affinity of the F11 Fab fragment to the HA ectodomain. (C,D) Results on F11 mutants used for the experimental mutagenesis. 


\subsection{Experimental Mutagenesis}

To assess the predictive validity of the in silico mutagenesis, we conducted an experimental mutagenesis study (Figure 6). We assumed that an increase in binding affinity by a given substitution might increase the neutralization activity of F11 against not only the original A/Narita/1/2009 (H1N1)pdm09 virus but also its variants. In this regard, a study on neutralization-resistant viruses might have virological significance. Therefore, we included two A/Narita/1/2009 (H1N1)pdm09 variants here, C1 and G6, that possess single mutations in the HA stalk domain and exhibit different levels of reduced sensitivity to the F11 IgG antibody: $\mathrm{C} 1$ has a highly resistant phenotype and G6 has a weakly resistant phenotype [18]. The $\mathrm{C} 1$ virus possessed a T333K substitution that has been reported to be involved in resistance to a broad range of anti-HA stalk antibodies in infection of MDCK cells by the A/Netherlands/602/2009 (H1N1)pdm09 virus [10].

A

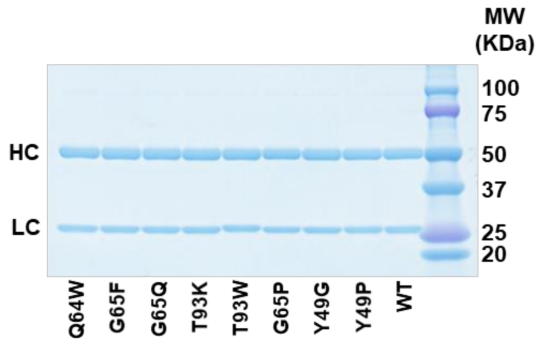

F11 antibody mutants

B

A/Narita/1/2009 (H1N1)pdm09

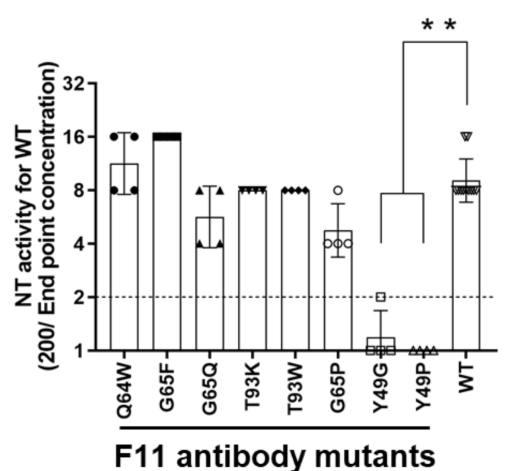

C1

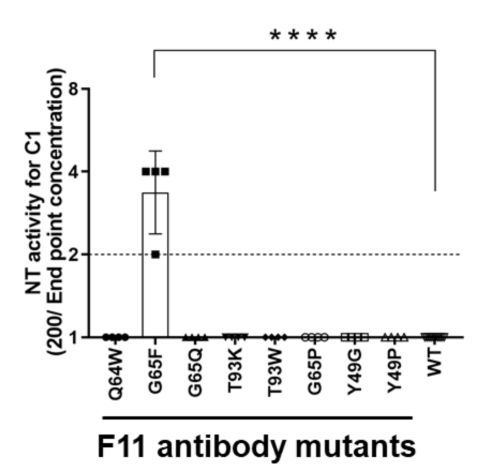

G6

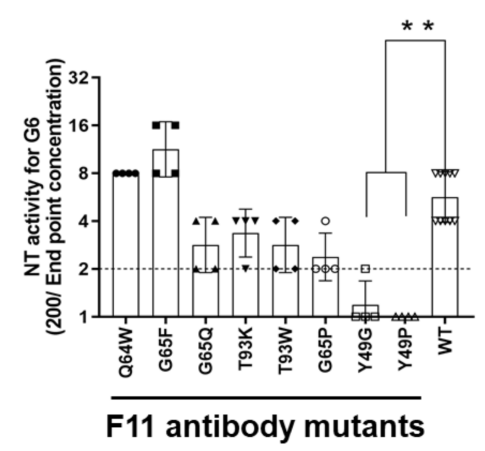

Figure 6. Experimental mutagenesis. Single amino acid substitutions were introduced into the Fab fragment of the F11 IgG1 antibody according to the information in Figure 5C,D. Affinity-purified soluble IgG1 antibodies were used to assess neutralization activity using humanized MDCK cells and $100 \mathrm{TCID}_{50}$ of influenza virus as described previously [48,49]. Neutralization activity was defined as the reciprocal of the lowest concentration $(\mu \mathrm{g} / \mathrm{mL})$ of antibody at which A630 was $>\mathrm{X}$ as calculated using the formula $\mathrm{X}=$ (A cell $-\mathrm{A}$ virus) $/ 2$. (A) SDS PAGE of the purified F11 IgG fractions used for the neutralization assay. (B) Neutralization activity against the F11-sensitive (H1N1)pdm09 virus [18] (left panel), F11-resistant (H1N1)pdm09 variant C1 [18] (middle panel), and F11-weakly resistant (H1N1)pdm09 variant G6 [18] (right panel). NT activity is presented in the scatter plots as the geometric mean, with the geometric standard deviation from four technical replicates (except for F11 WT: $n=10$ ). Y-axis values represent neutralizing titers, calculated as $100 /$ minimum concentration $(\mu \mathrm{g} / \mathrm{mL})$ of antibody that neutralized the virus. Dotted lines represent the detection limit $(\mathrm{y}=1 ; 100 \mu \mathrm{g} / \mathrm{mL}) .{ }^{* *} p<0.01$ and $* * * * p<0.0001$, comparing F11 mutants with F11 WT (Kruskal-Wallis test, followed by Dunn's multiple comparison test). For statistical analysis, a provisional minimum NT activity value $(y=0.5 ; 200 \mu \mathrm{g} / \mathrm{mL})$ was applied to samples in which NT activity was below the detection limit.

Purified IgG1 fractions of F11-derived mutants were prepared as described previously [18] and used for a neutralization assay with humanized MDCK cells $[48,49]$. We 
confirmed successful production and purification of F11 IgG1 variant antibodies by SDSPAGE (Figure 6A), as expected from in silico data on the stability of F11 Fab mutants (Figure 5C). Individual F11 mutations appeared to have distinct effects on the neutralization activity against the A/Narita/1/2009 (H1N1)pdm09 virus (Figure 6B, left panel). G65F in the F11 heavy chain, which was predicted to achieve the greatest increase in the binding affinity to HA (Figure 5D), consistently increased the neutralization activity of F11 by about two-fold, although statistical significance was not obtained (Figure 6B, G65F in the left panel). More remarkably, single substitutions at position 49 in the F11 light chain, which was predicted to moderately reduce the F11 binding affinity to HA (Figure 5D), consistently reduced F11 neutralization activity (Figure 6B, Y49G/P in the left panel). No statistically significant changes were detected with the other F11 mutants.

As expected from the previous study [18], the purified F11 IgG1 fraction had little neutralization activity against the F11-resistant $\mathrm{C} 1$ virus (Figure $6 \mathrm{~B}, \mathrm{WT}$ in the middle panel). Most of the F11 single substitutions also had little activity. Notably, however, G65F substitution was found to confer an important new phenotype on F11 IgG1: neutralization activity against the $\mathrm{C} 1$ virus (Figure 6B, G65F in the middle panel. The G65F substitution also had positive effects on activity against the G6 virus, as it did against the original virus (Figure 6B, G65F in the left and right panels). Here again, a marked reduction in neutralization activity was detected with F11 possessing Y49G/P substitutions. No statistically significant changes were detected with the other F11 mutants. Thus, the in silico data on the binding affinity, i.e., F11 substitutions exhibiting the greatest positive and negative effects on in silico binding affinity, were consistent with the positive and negative effects on neutralization activity.

\section{Discussion}

In this study, we characterized the molecular interactions between an anti-HA stalk antibody F11 Fab fragment and the trimeric form of the influenza A(H1N1)pdm09 HA glycoprotein. We then used this information to produce F11 variants with new phenotypes by single substitutions in the F11 variable region. Thus far, there has been only a single report on the structure-based creation of new antibodies against influenza viruses [65]. That earlier study presented a successful case of modification of an anti-neuraminidase antibody. To our knowledge, however, the present study is the first report to describe the creation of an anti-HA stalk antibody possessing neutralization activity against a neutralization-resistant virus.

In this study, we have constructed and characterized 100 complex models of an HA ectodomain docked to an F11 Fab fragment using homology modeling, MD simulation, and docking simulation to understand the structural basis of the actions and modifications of this antibody. Our in silico study with the 100 models disclosed that the F11 Fab fragment has physicochemical features that crosslink two HA monomers by binding to a region near the proteolytic cleavage site of the stalk domain (Figure 1). This unique binding mode is represented by the top-2 docking pose with the lowest free energy of binding and stability during MD simulation (Figure 2). Variation in the HA residues contacting the F11 Fab in this binding mode explains the neutralization specificity of the F11 antibody [18] (Figure 3) well. Moreover, the F11 Fab binding to HA was predicted to have little effect on the structural dynamics of the surfaces on the HA head domain for the sialic acid binding (Figure 4), consistent with our previous observation that the F11 antibody did not suppress the hemagglutination inhibition activity of HA [18]. Finally, F11 derivatives exhibiting positive and negative effects on neutralization activity were effectively obtained with the top-2 binding mode (Figures 5 and 6). Collectively, these results strongly suggest that the binding mode proposed in this study represents a physicochemically, thermodynamically, and virologically reasonable mode for the F11 Fab fragment. To date, similar binding modes involving the crosslinking of two HA monomers have often been reported with anti-HA stalk antibodies $[9,12-14,51,52]$. Thus, the crosslinking is likely to represent a favorable binding mode for the anti-HA stalk antibodies in general. 
Results of our present in silico studies suggest that there are at least two types of structural impacts for the F11-mediated neutralization of influenza viruses. First, the F11 binding to HA may disturb the cleavage of HA that is a prerequisite for the ordered conformational rearrangement to establish virus infection of the cells [11,60]. Our study suggests that F11 Fab can bind to a region near the proteolytic cleavage site of the stalk domain (Figure 3) and suppress the dynamics of structural units, such as the cleavage loop, proteolytic cleavage site, and fusion peptide (Figure 4). Because the structural dynamics of the molecular interaction surface is critical for the molecular interactions and thus the function of biomolecules [18,31-33,55-58], it is possible that F11 binding not only sterically but also physically inhibits the proteolysis of HA by cellular proteases. In this regard, it is worth noting that proteolysis of HA is suggested to occur with proteases in human respiratory epithelial cells $[59,66]$. Such proteolysis can occur with HA on the virion by the cell surface human airway trypsin-like protease $[59,66]$ prior to endocytosis. Therefore, F11 binding to HA on the incoming virion outside of the cells may be able to disturb the proteolytic cleavage during virus entry and consequently block initiation of the conformational rearrangement of HA in the cells.

Alternatively, the F11 binding to HA may inhibit pH-dependent conformational changes in the endosomes that are required for viral membrane fusion and genome uncoating in the cells $[11,50,61,67]$. Our in silico study suggests that the F11 Fab fragment can crosslink two HA monomers by binding to a conserved region near the proteolytic cleavage site of the stalk domain (Figure 3 ). This binding mode should sterically prevent the conformational changes in endosomes, such as exposure of the fusion peptide, and thus block subsequent processes, such as membrane fusion and genome uncoating, in the case that F11 maintained its binding ability to HA under acidic conditions. Further study is necessary to address each of these possibilities.

Next, we attempted to modify F11 based on the structural information related to the F11 Fab-trimeric HA ectodomain interactions. Structure-guided mutagenesis of the F11 antibody led us to identification of a residue 49 in the F11 light chain as a critical site for the F11-mediated neutralization (Figures 5 and 6). Single amino acid substitutions at this site almost completely abolished the neutralization activity of F11. Reduction in the binding affinity was constantly detected for each of the complexes during the MD simulations, suggesting the affinity reduction could occur in solution (Figure S3). However, it is hard to explain the complete abolishment of F11 neutralization activity by affinity reduction alone because the levels of reduction were rather small, and because considerable affinities remained during the MD simulation (Figure S3). Therefore, it is likely that additional factors in addition to the affinity reduction are involved in the dysfunction of F11 possessing the single substitutions at position 49. In this regard, it should be noted that the F11 residue at position 49 presumably interacts noncovalently with an HA glycan moiety on asparagine at position 40 (N40) near the HA cleavage site by forming a hydrogen bond, and that a single substitution at position 49 of F11 could result in the loss of this interaction (Figure S3). These results suggest that the free glycan moiety on N40 is critical for virus infection of cells. Nonetheless, the function of this glycan in viral protection against the anti-HA stalk antibody [14,68], as well as its function in virus infection and replication have not been reported thus far. Further study on the function of this particular glycan near the HA cleavage site may provide new insights into the mechanisms of influenza virus infection and replication. It is important to study such resistance mutations and develop methods to overcome the resistance, because antibodies targeting the stalk domain can be a powerful tool to combat infections of influenza viruses [6-8].

We previously isolated two $\mathrm{A}(\mathrm{H} 1 \mathrm{~N} 1) \mathrm{pdm} 09$ variants, the $\mathrm{C} 1$ and $\mathrm{G} 6$ viruses, that exhibited potent and weak resistance to F11, respectively [18]. Interestingly, a recent study has shown that T333K, a single substitution detected in the $\mathrm{C} 1$ virus, is involved in resistance to a broad range of anti-HA stalk antibodies [9,10], indicating that the residue is a key player for the anti-HA stalk antibody resistance. Curiously, a virus possessing the T333K substitution still retained an ability to bind to the anti-HA stalk antibodies FI6 [10]. 
The results indicate that the T333K substitution functions in the resistance by mechanisms that are not involved in the prevention of antibody binding. One such mechanism may be the T333K-induced restoration of dysregulated structural dynamics of HA caused by antibody binding. In this study, we have suggested by MD simulation that F11 antibody binding to the trimeric HA induces dysregulation of structural dynamics in the fusion loop and its flanking regions (Figure 4). This structural defect may be restored by the T333K substitution, so that proteolytic cleavage proceeds to initiate conformational changes for infection. Further studies involving MD simulation will help to address this issue.

Our structure-guided mutagenesis of the F11 antibody in this study led to identification of an F11 derivative that suppresses infections by the C1 and G6 viruses (Figures 5 and 6). Importantly, G65F single substitution in the F11 heavy chain was sufficient to overcome the resistance (Figure 6B). At present, the molecular mechanism underlying this phenomenon remains unclear. A possible mechanism might be a dysfunction of the HA moieties by G65F substitution. The F11 residue at position 65 in the heavy chain presumably contacts the amino acid and glycan residues near the hydrophobic groove (Figure 3A,B). Thus far, the functions of these HA moieties in virus infection and replication have not been reported. Interestingly, the G65F substitution was suggested to induce drastic changes in the interaction networks around R62, K319, and M403 (Figure S3B), which may suppress the structural dynamics around these residues. Further structure-functional studies on these particular amino acids and glycan residues may provide new insights into the mechanisms of influenza virus infection and replication for antibody modification.

In conclusion, we here proposed a physicochemically, thermodynamically, and virologically reasonable model of the anti-HA stalk antibody F11 and trimeric HA ectodomain. The model involves crosslinking of two HA monomers and has provided new clues for understanding the structural mechanisms of virus neutralization by the F11 anti-HA stalk antibody. Moreover, the model was found to be useful to identify a critical site for the F11-mediated neutralization, as well as to generate anti-HA stalk antibodies that acquired the ability to suppress infections of F11-resistant viruses. Since the anti-HA stalk antibody is a potential tool to combat the influenza virus [6-8], it would be important to understand the mechanisms that underlie the neutralization of neutralization-resistant viruses using the anti-HA stalk antibody derivative obtained in this study.

Supplementary Materials: The following are available online at https:/ / www.mdpi.com/article / 10.3390/v13091733/s1: Figure S1: Amino acid sequences of the F11 Fab fragment and HA protein of the influenza A/Narita/1/2009 (H1N1)pdm09 strain; Figure S2: Binding modes in the docking simulations of the F11 Fab fragment and glycosylated HA trimer of influenza A/Narita/1/2009 (H1N1)pdm09; Figure S3: Effects of the F11 Y49P and G65F substitutions on molecular interactions.

Author Contributions: Conceptualization, O.K. and S.S.; methodology, O.K., Y.S., A.A. and S.S.; software, O.K. and M.Y.; validation, O.K., Y.S., A.A., M.Y., K.S., K.T. and S.S.; formal analysis, O.K., Y.S., A.U., T.H. and S.S.; investigation, O.K., Y.S., A.U., T.H. and S.S.; resources, O.K., Y.S., K.S., K.T., T.S., H.H. and S.S.; data curation, O.K., Y.S. and S.S.; writing-original draft preparation, O.K., S.S. and H.S.; writing-review and editing, H.S., O.K., Y.S., S.S., A.A., A.U., T.H., K.S., K.T., M.Y., T.S. and H.H.; visualization, O.K. and S.S.; supervision, T.S., H.H. and H.S.; project administration, O.K. and S.S.; funding acquisition, O.K., S.S., A.A. and H.S. All authors have read and agreed to the published version of the manuscript.

Funding: This work was supported in part by grants from the Japan Agency for Medical Research and Development, AMED (Research Program on Emerging and Re-emerging Infectious Diseases: JP20fk0108107 and JP21fk0108107 to OK, AA, and SS, and JP20fk0108118 and JP21fk0108118 to HS).

Institutional Review Board Statement: Not applicable.

Informed Consent Statement: Not applicable.

Data Availability Statement: Please contact the corresponding authors (O.K. and S.S.) for data on the present study. 
Acknowledgments: The MD simulations were partly carried out with the TSUBAME3.0 supercomputer at the Tokyo Institute of Technology. We thank Hiromi Nakamura and Akiko Sataka for their technical assistance.

Conflicts of Interest: The authors declare no conflict of interest. The funders had no role in the design of the study; in the collection, analyses, or interpretation of data; in the writing of the manuscript; or in the decision to publish the results.

$\begin{array}{ll}\text { Abbreviations } \\ \text { CDR } & \begin{array}{l}\text { complementarity determining region } \\ \text { constant region }\end{array} \\ \text { CR } & \text { Dulbecco's Modified Eagle's Medium } \\ \text { DMEM } & \text { Extended Huckel Theory } \\ \text { EHT } & \text { Hemagglutinin } \\ \text { HA } & \text { Humanized MDCK } \\ \text { hCK } & \text { Molecular dynamics } \\ \text { MD } & \text { Madin-Darby canine kidney } \\ \text { MDCK } & \text { Minimum essential medium } \\ \text { MEM } & \text { Molecular Operating Environment } \\ \text { MOE } & \text { microneutralization } \\ \text { NT } & \text { Root mean square deviation } \\ \text { RMSD } & \text { Root mean square fluctuation } \\ \text { RMSF } & \text { variable region }\end{array}$

\section{References}

1. Altman, M.O.; Angeletti, D.; Yewdell, J.W. Antibody immunodominance: The key to understanding influenza virus antigenic drift. Viral Immunol. 2018, 31, 142-149. [CrossRef]

2. Wu, N.C.; Wilson, I.A. A perspective on the structural and functional constraints for immune evasion: Insights from influenza virus. J. Mol. Biol. 2017, 429, 2694-2709. [CrossRef]

3. Bhatt, S.; Holmes, E.C.; Pybus, O.G. The genomic rate of molecular adaptation of the human influenza a virus. Mol. Biol. Evol. 2011, 28, 2443-2451. [CrossRef] [PubMed]

4. Kirkpatrick, E.; Qiu, X.; Wilson, P.C.; Bahl, J.; Krammer, F. The influenza virus hemagglutinin head evolves faster than the stalk domain. Sci. Rep. 2018, 8, 10432. [CrossRef] [PubMed]

5. Webster, R.G.; Bean, W.J.; Gorman, O.T.; Chambers, T.M.; Kawaoka, Y. Evolution and ecology of influenza a viruses. Microbiol. Rev. 1992, 56, 152-179. [CrossRef] [PubMed]

6. Coughlan, L.; Palese, P. Overcoming barriers in the path to a universal influenza virus vaccine. Cell Host Microbe 2018, 24 , 18-24. [CrossRef] [PubMed]

7. Stadlbauer, D.; Nachbagauer, R.; Meade, P.; Krammer, F. Universal influenza virus vaccines: What can we learn from the human immune response following exposure to $\mathrm{H7}$ subtype viruses? Front. Med. 2017, 11, 471-479. [CrossRef]

8. Sedeyn, K.; Saelens, X. New antibody-based prevention and treatment options for influenza. Antivir. Res. 2019, 170, 104562. [CrossRef]

9. Corti, D.; Voss, J.; Gamblin, S.J.; Codoni, G.; Macagno, A.; Jarrossay, D.; Vachieri, S.G.; Pinna, D.; Minola, A.; Vanzetta, F.; et al. A neutralizing antibody selected from plasma cells that binds to group 1 and group 2 influenza A hemagglutinins. Science 2011, 333, 850-856. [CrossRef]

10. Roubidoux, E.K.; Carreno, J.M.; McMahon, M.; Jiang, K.; van Bakel, H.; Wilson, P.; Krammer, F. Mutations in the hemagglutinin stalk domain do not permit escape from a protective, stalk-based vaccine-induced immune response in the mouse model. $m B i o$ 2021, 12, e03617-20. [CrossRef]

11. Benton, D.J.; Gamblin, S.J.; Rosenthal, P.B.; Skehel, J.J. Structural transitions in influenza haemagglutinin at membrane fusion pH. Nature 2020, 583, 150-153. [CrossRef]

12. Joyce, M.G.; Wheatley, A.K.; Thomas, P.V.; Chuang, G.Y.; Soto, C.; Bailer, R.T.; Druz, A.; Georgiev, I.S.; Gillespie, R.A.; Kanekiyo, M.; et al. Vaccine-Induced antibodies that neutralize group 1 and group 2 influenza a viruses. Cell 2016, 166, 609-623. [CrossRef]

13. Wu, Y.; Cho, M.; Shore, D.; Song, M.; Choi, J.; Jiang, T.; Deng, Y.Q.; Bourgeois, M.; Almli, L.; Yang, H.; et al. A potent broadspectrum protective human monoclonal antibody crosslinking two haemagglutinin monomers of influenza A virus. Nat. Commun. 2015, 6, 7708. [CrossRef] [PubMed]

14. Ekiert, D.C.; Bhabha, G.; Elsliger, M.A.; Friesen, R.H.; Jongeneelen, M.; Throsby, M.; Goudsmit, J.; Wilson, I.A. Antibody recognition of a highly conserved influenza virus epitope. Science 2009, 324, 246-251. [CrossRef]

15. Dreyfus, C.; Laursen, N.S.; Kwaks, T.; Zuijdgeest, D.; Khayat, R.; Ekiert, D.C.; Lee, J.H.; Metlagel, Z.; Bujny, M.V.; Jongeneelen, M.; et al. Highly conserved protective epitopes on influenza b viruses. Science 2012, 337, 1343-1348. [CrossRef] 
16. Kallewaard, N.L.; Corti, D.; Collins, P.J.; Neu, U.; McAuliffe, J.M.; Benjamin, E.; Wachter-Rosati, L.; Palmer-Hill, F.J.; Yuan, A.Q.; Walker, P.A.; et al. Structure and function analysis of an antibody recognizing all influenza a subtypes. Cell 2016, 166, 596-608. [CrossRef]

17. Lang, S.; Xie, J.; Zhu, X.; Wu, N.C.; Lerner, R.A.; Wilson, I.A. Antibody 27F3 broadly targets influenza a group 1 and 2 hemagglutinins through a further variation in VH1-69 antibody orientation on the HA stem. Cell Rep. 2017, 20, 2935-2943. [CrossRef]

18. Sano, K.; Saito, S.; Suzuki, T.; Kotani, O.; Ainai, A.; van Riet, E.; Tabata, K.; Saito, K.; Takahashi, Y.; Yokoyama, M.; et al. An influenza HA stalk reactive polymeric IgA antibody exhibits anti-viral function regulated by binary interaction between $\mathrm{HA}$ and the antibody. PLoS ONE 2021, 16, e0245244. [CrossRef]

19. Ainai, A.; Hasegawa, H.; Obuchi, M.; Odagiri, T.; Ujike, M.; Shirakura, M.; Nobusawa, E.; Tashiro, M.; Asanuma, H. Host adaptation and the alteration of viral properties of the first influenza A/H1N1pdm09 virus isolated in Japan. PLoS ONE 2015, 10, e0130208. [CrossRef]

20. Xu, R.; Ekiert, D.C.; Krause, J.C.; Hai, R.; Crowe, J.E., Jr.; Wilson, I.A. Structural basis of preexisting immunity to the 2009 H1N1 pandemic influenza virus. Science 2010, 328, 357-360. [CrossRef] [PubMed]

21. GLYCAM-Web. Available online: http:/ / glycam.org (accessed on 1 March 2018).

22. Yokoyama, M.; Fujisaki, S.; Shirakura, M.; Watanabe, S.; Odagiri, T.; Ito, K.; Sato, H. Molecular dynamics simulation of the influenza $\mathrm{A}(\mathrm{H} 3 \mathrm{~N} 2)$ hemagglutinin trimer reveals the structural basis for adaptive evolution of the recent epidemic clade 3C.2a. Front. Microbiol. 2017, 8, 584. [CrossRef]

23. Case, D.A.; Betz, R.M.; Cerutti, D.S.; Cheatham, T.E., III; Darden, T.A.; Duke, R.E.; Giese, T.J.; Gohlke, H.; Goetz, A.W.; Homeyer, N.; et al. AMBER 16; University of California: San Francisco, CA, USA, 2016.

24. Maier, J.A.; Martinez, C.; Kasavajhala, K.; Wickstrom, L.; Hauser, K.E.; Simmerling, C. ff14SB: Improving the accuracy of protein side chain and backbone parameters from ff99SB. J. Chem. Theory Comput. 2015, 11, 3696-3713. [CrossRef]

25. Kirschner, K.N.; Yongye, A.B.; Tschampel, S.M.; Gonzalez-Outeirino, J.; Daniels, C.R.; Foley, B.L.; Woods, R.J. GLYCAM06: A generalizable biomolecular force field. Carbohydrates. J. Comput. Chem. 2008, 29, 622-655. [CrossRef]

26. Jorgensen, W.L.; Chandrasekhar, J.; Madura, J.D.; Impei, R.W.; Klein, M.L. Comparison of simple potential functions for simulating liquid water. J. Chem. Phys. 1983, 79, 926-935. [CrossRef]

27. Ryckaert, J.-P.; Ciccotti, G.; Berendsen, H.J.C. Numerical integration of the cartesian equations of motion of a system with constraints: Molecular dynamics of n-alkanes. J. Comput. Phys. 1977, 23, 327-341. [CrossRef]

28. Maier, J.K.; Labute, P. Assessment of fully automated antibody homology modeling protocols in molecular operating environment. Proteins 2014, 82, 1599-1610. [CrossRef]

29. Pagadala, N.S.; Syed, K.; Tuszynski, J. Software for molecular docking: A review. Biophys. Rev. 2017, 9, 91-102. [CrossRef]

30. Corbeil, C.R.; Williams, C.I.; Labute, P. Variability in docking success rates due to dataset preparation. J. Comput.-Aided Mol. Des. 2012, 26, 775-786. [CrossRef] [PubMed]

31. Koma, T.; Kotani, O.; Miyakawa, K.; Ryo, A.; Yokoyama, M.; Doi, N.; Adachi, A.; Sato, H.; Nomaguchi, M. Allosteric regulation of HIV-1 capsid structure for gag assembly, virion production, and viral infectivity by a disordered interdomain linker. J. Virol. 2019, 93, e00381-19. [CrossRef]

32. Koma, T.; Yokoyama, M.; Kotani, O.; Doi, N.; Nakanishi, N.; Okubo, H.; Adachi, S.; Adachi, A.; Sato, H.; Nomaguchi, M. Species-Specific Valid ternary interactions of HIV-1 Env-gp120, CD4, and CCR5 as revealed by an adaptive single-amino acid substitution at the V3 loop tip. J. Virol. 2021, 95, e0217720. [CrossRef]

33. Sakuragi, S.; Kotani, O.; Yokoyama, M.; Shioda, T.; Sato, H.; Sakuragi, J.I. Identification of a novel cis-acting regulator of HIV-1 genome packaging. Int. J. Mol. Sci. 2021, 22, 3435. [CrossRef]

34. Shannon, P.; Markiel, A.; Ozier, O.; Baliga, N.S.; Wang, J.T.; Ramage, D.; Amin, N.; Schwikowski, B.; Ideker, T. Cytoscape: A software environment for integrated models of biomolecular interaction networks. Genome Res. 2003, 13, 2498-2504. [CrossRef]

35. Capponi, S.; Geuens, T.; Geroldi, A.; Origone, P.; Verdiani, S.; Cichero, E.; Adriaenssens, E.; De Winter, V.; Bandettini di Poggio, M.; Barberis, M.; et al. Molecular chaperones in the pathogenesis of amyotrophic lateral sclerosis: The role of HSPB1. Hum. Mutat. 2016, 37, 1202-1208. [CrossRef]

36. Dindo, M.; Montioli, R.; Busato, M.; Giorgetti, A.; Cellini, B.; Borri Voltattorni, C. Effects of interface mutations on the dimerization of alanine glyoxylate aminotransferase and implications in the mistargeting of the pathogenic variants F152I and I244T. Biochimie 2016, 131, 137-148. [CrossRef]

37. Jetha, A.; Thorsteinson, N.; Jmeian, Y.; Jeganathan, A.; Giblin, P.; Fransson, J. Homology modeling and structure-based design improve hydrophobic interaction chromatography behavior of integrin binding antibodies. MAbs 2018, 10, 890-900. [CrossRef]

38. Shah, M.; Anwar, M.A.; Park, S.; Jafri, S.S.; Choi, S. In silico mechanistic analysis of IRF3 inactivation and high-risk HPV E6 species-dependent drug response. Sci. Rep. 2015, 5, 13446. [CrossRef]

39. Agrawal, N.J.; Kumar, S.; Wang, X.; Helk, B.; Singh, S.K.; Trout, B.L. Aggregation in protein-based biotherapeutics: Computational studies and tools to identify aggregation-prone regions. J. Pharm. Sci. 2011, 100, 5081-5095. [CrossRef]

40. Chennamsetty, N.; Voynov, V.; Kayser, V.; Helk, B.; Trout, B.L. Design of therapeutic proteins with enhanced stability. Proc. Natl. Acad. Sci. USA 2009, 106, 11937-11942. [CrossRef] 
41. Motomura, K.; Oka, T.; Yokoyama, M.; Nakamura, H.; Mori, H.; Ode, H.; Hansman, G.S.; Katayama, K.; Kanda, T.; Tanaka, T.; et al. Identification of monomorphic and divergent haplotypes in the 2006-2007 norovirus GII/4 epidemic population by genomewide tracing of evolutionary history. J. Virol. 2008, 82, 11247-11262. [CrossRef]

42. GISAID. Available online: https:/ / www.gisaid.org. (accessed on 1 June 2018).

43. Shannon, C.E. The mathematical theory of communication. 1963. MD Comput. 1997, 14, 306-317.

44. Takashita, E.; Fujisaki, S.; Yokoyama, M.; Shirakura, M.; Morita, H.; Nakamura, K.; Kishida, N.; Kuwahara, T.; Sato, H.; Doi, I.; et al. In Vitro characterization of multidrug-resistant influenza A(H1N1)pdm09 viruses carrying a dual neuraminidase mutation isolated from immunocompromised patients. Pathogens 2020, 9, 725. [CrossRef]

45. Takashita, E.; Kiso, M.; Fujisaki, S.; Yokoyama, M.; Nakamura, K.; Shirakura, M.; Sato, H.; Odagiri, T.; Kawaoka, Y.; Tashiro, M. Characterization of a large cluster of influenza A(H1N1)pdm09 viruses cross-resistant to oseltamivir and peramivir during the 2013-2014 influenza season in Japan. Antimicrob. Agents Chemother. 2015, 59, 2607-2617. [CrossRef]

46. Nomaguchi, M.; Yokoyama, M.; Kono, K.; Nakayama, E.E.; Shioda, T.; Doi, N.; Fujiwara, S.; Saito, A.; Akari, H.; Miyakawa, K.; et al. Generation of rhesus macaque-tropic HIV-1 clones that are resistant to major anti-HIV-1 restriction factors. J. Virol. 2013, 87, 11447-11461. [CrossRef]

47. Takada, K.; Kawakami, C.; Fan, S.; Chiba, S.; Zhong, G.; Gu, C.; Shimizu, K.; Takasaki, S.; Sakai-Tagawa, Y.; Lopes, T.J.S.; et al. A humanized MDCK cell line for the efficient isolation and propagation of human influenza viruses. Nat. Microbiol. 2019, 4, 1268-1273. [CrossRef]

48. Ainai, A.; Tamura, S.; Suzuki, T.; van Riet, E.; Ito, R.; Odagiri, T.; Tashiro, M.; Kurata, T.; Hasegawa, H. Intranasal vaccination with an inactivated whole influenza virus vaccine induces strong antibody responses in serum and nasal mucus of healthy adults. Hum. Vaccin. Immunother. 2013, 9, 1962-1970. [CrossRef]

49. Saito, S.; Sano, K.; Suzuki, T.; Ainai, A.; Taga, Y.; Ueno, T.; Tabata, K.; Saito, K.; Wada, Y.; Ohara, Y.; et al. IgA tetramerization improves target breadth but not peak potency of functionality of anti-influenza virus broadly neutralizing antibody. PLoS Pathog. 2019, 15, e1007427. [CrossRef]

50. Knipe, D.M.; Howley, P.M. Orthomyxoviridae. Fields Virol. 2013, 1, 1151-1185.

51. Harshbarger, W.D.; Deming, D.; Lockbaum, G.J.; Attatippaholkun, N.; Kamkaew, M.; Hou, S.; Somasundaran, M.; Wang, J.P.; Finberg, R.W.; Zhu, Q.K.; et al. Unique structural solution from a VH3-30 antibody targeting the hemagglutinin stem of influenza a viruses. Nat. Commun. 2021, 12, 559. [CrossRef] [PubMed]

52. Wu, N.C.; Yamayoshi, S.; Ito, M.; Uraki, R.; Kawaoka, Y.; Wilson, I.A. Recurring and adaptable binding motifs in broadly neutralizing antibodies to influenza virus are encoded on the D3-9 segment of the ig gene. Cell Host Microbe 2018, 24, 569-578.e4 [CrossRef]

53. Dreyfus, C.; Ekiert, D.C.; Wilson, I.A. Structure of a classical broadly neutralizing stem antibody in complex with a pandemic H2 influenza virus hemagglutinin. J. Virol. 2013, 87, 7149-7154. [CrossRef] [PubMed]

54. Nath Neerukonda, S.; Vassell, R.; Weiss, C.D. Neutralizing antibodies targeting the conserved stem region of influenza hemagglutinin. Vaccines 2020, 8, 382. [CrossRef] [PubMed]

55. Bai, H.; Kath, J.E.; Zorgiebel, F.M.; Sun, M.; Ghosh, P.; Hatfull, G.F.; Grindley, N.D.; Marko, J.F. Remote control of DNA-acting enzymes by varying the brownian dynamics of a distant DNA end. Proc. Natl. Acad. Sci. USA 2012, 109, 16546-16551. [CrossRef] [PubMed]

56. Dodson, G.G.; Lane, D.P.; Verma, C.S. Molecular simulations of protein dynamics: New windows on mechanisms in biology. EMBO Rep. 2008, 9, 144-150. [CrossRef]

57. Karplus, M.; Kuriyan, J. Molecular dynamics and protein function. Proc. Natl. Acad. Sci. USA 2005, 102, 6679-6685. [CrossRef]

58. Keul, N.D.; Oruganty, K.; Schaper Bergman, E.T.; Beattie, N.R.; McDonald, W.E.; Kadirvelraj, R.; Gross, M.L.; Phillips, R.S.; Harvey, S.C.; Wood, Z.A. The entropic force generated by intrinsically disordered segments tunes protein function. Nature 2018, 563, 584-588. [CrossRef]

59. Bottcher, E.; Matrosovich, T.; Beyerle, M.; Klenk, H.D.; Garten, W.; Matrosovich, M. Proteolytic activation of influenza viruses by serine proteases TMPRSS2 and HAT from human airway epithelium. J. Virol. 2006, 80, 9896-9898. [CrossRef]

60. Callan, R.J.; Hartmann, F.A.; West, S.E.; Hinshaw, V.S. Cleavage of influenza a virus H1 hemagglutinin by swine respiratory bacterial proteases. J. Virol. 1997, 71, 7579-7585. [CrossRef]

61. Hamilton, B.S.; Whittaker, G.R.; Daniel, S. Influenza virus-mediated membrane fusion: Determinants of hemagglutinin fusogenic activity and experimental approaches for assessing virus fusion. Viruses 2012, 4, 1144-1168. [CrossRef]

62. Stieneke-Grober, A.; Vey, M.; Angliker, H.; Shaw, E.; Thomas, G.; Roberts, C.; Klenk, H.D.; Garten, W. Influenza virus hemagglutinin with multibasic cleavage site is activated by furin, a subtilisin-like endoprotease. EMBO J. 1992, 11, 2407-2414. [CrossRef] [PubMed]

63. Sun, X.; Shi, Y.; Lu, X.; He, J.; Gao, F.; Yan, J.; Qi, J.; Gao, G.F. Bat-Derived influenza hemagglutinin H17 does not bind canonical avian or human receptors and most likely uses a unique entry mechanism. Cell Rep. 2013, 3, 769-778. [CrossRef] [PubMed]

64. Sriwilaijaroen, N.; Suzuki, Y. Molecular basis of the structure and function of H1 hemagglutinin of influenza virus. Proc. Jpn. Acad. Ser. B Phys. Biol. Sci. 2012, 88, 226-249. [CrossRef]

65. Jiang, H.; Peng, W.; Qi, J.; Chai, Y.; Song, H.; Bi, Y.; Rijal, P.; Wang, H.; Oladejo, B.O.; Liu, J.; et al. Structure-Based modification of an anti-neuraminidase human antibody restores protection efficacy against the drifted influenza virus. mBio 2020, 11, e02315-20. [CrossRef] [PubMed] 
66. Bottcher-Friebertshauser, E.; Freuer, C.; Sielaff, F.; Schmidt, S.; Eickmann, M.; Uhlendorff, J.; Steinmetzer, T.; Klenk, H.D.; Garten, W. Cleavage of influenza virus hemagglutinin by airway proteases TMPRSS2 and HAT differs in subcellular localization and susceptibility to protease inhibitors. J. Virol. 2010, 84, 5605-5614. [CrossRef]

67. Dou, D.; Revol, R.; Ostbye, H.; Wang, H.; Daniels, R. Influenza a virus cell entry, replication, virion assembly and movement. Front. Immunol. 2018, 9, 1581. [CrossRef]

68. Liu, W.C.; Jan, J.T.; Huang, Y.J.; Chen, T.H.; Wu, S.C. Unmasking stem-specific neutralizing epitopes by abolishing n-linked glycosylation sites of influenza virus hemagglutinin proteins for vaccine design. J. Virol. 2016, 90, 8496-8508. [CrossRef] 